\title{
CONSTRUCCIÓN PARTICIPATIVA DE LA FORMULACIÓN DE LA POLÍTICA PÚBLICA DE GENERACIÓN DE EMPLEO De Puerto Boyacá
}

\author{
Richard Walter Triana* \\ Manuelita Barrios Rodríguez ${ }^{* *}$ \\ Carlos Ariel Rodríguez Vergara ${ }^{* * *}$
}

\section{Resumen}

El empleo es uno de los ejes más importantes para el desarrollo local dado que, por un lado, dinamiza la economía a través de la circulación del dinero que reciben los empleados, y, por el otro, contribuye al bienestar del individuo y su núcleo familiar. Es por ello que el desempleo es uno de los mayores retos que afronta cualquier Gobierno, en especial en la construcción de políticas que generen oportunidades de acceso al trabajo decente, de manera incluyente. Este artículo presenta la experiencia de construcción social de la política pública de generación de empleo de Puerto Boyacá (Boyacá), la cual se llevó a cabo desde un enfoque de desarrollo económico local y territorial, diferencial y trabajo decente.

Palabras clave: política pública, empleo, desarrollo local.

* Magíster en Pensamiento Estratégico y Prospectiva, Universidad Externado de Colombia (Colombia). Director Ejecutivo Centro de Estudios Regionales del Magdalena Medio (CER). [richard.triana@cer.org.co].

** Magíster en Políticas Públicas, Universidad Externado de Colombia (Colombia). Estudiante Doctorado en Ciencias Políticas, Administración y Relaciones Internacionales de la Universidad Complutense de Madrid (España). Profesional de Investigación Centro de Estudios Regionales del Magdalena Medio (CER). [manuelita.barrios@ cer.org.co].

*** Especialista en Proyectos de Desarrollo, Escuela Superior de Administración Pública (ESAP), Bogotá (Colombia). Profesional de Investigación Centro de Estudios Regionales del Magdalena Medio Cer. [carlosariel.rodriguez@cer. org.co].

Recibido: 8 de septiembre de 2017 / Modificado: 14 de diciembre de 2017 / Aceptado: 19 de enero de 2018.

Este artículo aporta a la solución del foco-reto país sociedad desarrollado en el marco del programa de Colombia Científica.

Para citar este artículo

Triana, R. W., Barrios Rodríguez, M. y Rodríguez Vergara, C. A. (2018). Construcción participativa de la formulación de la política pública de generación de empleo de Puerto Boyacá. opera, 22, pp. 29-51.

DoI: https://doi.org/10.18601/16578651.n22.03 


\section{PARTICIPATIVE FORMULATION OF THE EMPLOYMENT GENERATION POLICY IN PUERTO BOYACÁ}

\section{Abstract}

Employment is one of the most important axes for local development. On one hand, it dynamizes the economy through money circulation; on the other, it contributes to wellbeing of individuals and families. As a public problem, employment is always a challenge. Therefore governments aim at the construction of policies enabling opportunities for people to access to decent and inclusive work. This article discusses a facilitation process aiming at the collective formulation of the Employment Generation Policy in Puerto Boyacá (Boyacá), based on a local, differential and decent work approach.

Key words: Public policy, employment, local developed.

\section{INTRODUCCIÓN}

El desempleo es una de las problemáticas complejas que afronta el país, puesto que constituye gran parte de la base que dinamiza la economía y el bienestar de una comunidad. De otro lado, es uno de los factores determinantes para el desarrollo económico local y la inclusión social, que permite el sostenimiento del núcleo familiar, así como la activación de la economía, a través de la compra de bienes y servicios (González, Peiró, Bravo, 1996). Como señala Joseph Stiglitz (2002), "el empleo es importante para la cohesión social. La gente que no tiene trabajo está muy insatisfecha”.
Es por ello que uno de los mayores retos de la generación de puestos de trabajo es pasar de enfocarse en un proceso económico independiente de la satisfacción del trabajador, a uno que procure la obtención de un salario que garantice el bienestar de la persona. Por ejemplo, se han establecido políticas que incentivan el empleo a costa de salarios bajos, sin dimensionar si es lo más conveniente para incentivar la economía, o, como señala Keynes (1943), dejando de recibir el salario real, lo que puede generar el desempleo involuntario, es decir, personas que prefieren dejar de trabajar antes de recibir una menor remuneración a la que les corresponde. $\mathrm{Al}$ respecto, Stiglitz menciona:

Las políticas del pasado han engendrado un círculo vicioso: políticas macroeconómicas fallidas han llevado a un alto nivel de desempleo que a su vez ha conducido, o por lo menos contribuido, a la violencia urbana y la guerra de guerrillas; éstas, también a su vez, han desalentado la inversión e impedido el crecimiento (2003, p. 30).

En este sentido, el empleo debe estudiarse desde una perspectiva integral, que vaya más allá de datos estadísticos que indiquen su aumento o destrucción, y permita analizar el contexto social, económico y político; esto con el fin de contribuir a la concepción del desarrollo como transformación social. Para que esto se dé, Stiglitz (2002) plantea que se deben cumplir los siguientes objetivos: 1) aprender de los errores; 2) crear empleo; 3) un buen manejo de lo público; y 4) el fortalecimiento del conocimiento, que se debe ver reflejado en el mejoramiento de la calidad de la educación.

En respuesta a lo anterior emergen alternativas como la construcción de políticas públicas, las cuales nacen de la voluntad de 
los actores del territorio de dar solución a una o varias problemáticas que se han convertido en parte de la agenda pública, es decir, cuando pasan a ser un tema de interés que es reconocido e identificado tanto por las autoridades territoriales como por la comunidad en general. Como señala Roth: "Una política existe siempre y cuando las instituciones estatales asuman total o parcialmente la tarea de alcanzar objetivos estimados como deseables o necesarios, por medio de un proceso destinado a cambiar un estado de las cosas percibido como problemático" (2002, p. 27).

El proceso en el que un problema se inserta en la agenda pública se puede dar: 1) de arriba hacia abajo (top down), es decir, cuando los gobernantes consideran que un tema es importante; 2) de abajo hacia arriba (bottom $u p$ ), cuando los miembros de una comunidad visibilizan las problemáticas y utilizan diferentes mecanismos (p. ej. comunicados de prensa, vías de hecho) para lograr la atención de las autoridades públicas, así como una solución desde la gestión pública (programas, proyectos) (Roth, 2002; Mozas y Bernal, 2006; Parsons, 2013); 3) horizontal, cuando nace de un proceso de construcción social, en el que las autoridades locales, la comunidad y el sector privado identifican las problemáticas que pueden surgir alrededor de un tema de interés común y buscan alternativas de solución conjuntas.

En el presente artículo se esboza la experiencia de construcción social de la política pública de generación de empleo (PPGE) del municipio de Puerto Boyacá (Boyacá), se señalan los enfoques de abordaje utilizados, la metodología de construcción participativa, así como los aprendizajes obtenidos durante el proceso, dado que se considera una iniciativa que aporta al desarrollo territorial, tomando como eje principal el diálogo entre los actores del territorio para delimitar las acciones que generen bienestar social (figura 1).

\section{FIGURA 1. ENFOQUES DE LA FORMULACIÓN DE LA POLÍTICA PÚBLICA DE EMPLEO}

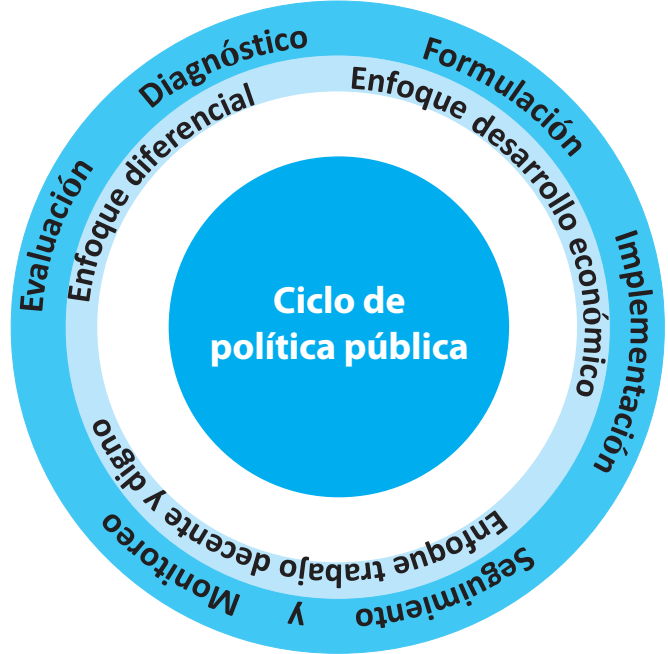

Fuente: CER (2017)

\section{ENFOQUES DE ABORDAJE DE LA FORMULACIÓN DE LA POLÍTICA PÚBLICA DE EMPLEO}

Los principales retos que afronta la realización de una política pública de empleo están relacionados con cerrar las brechas entre el mercado y la oferta laboral, mejorar la calidad del empleo y las oportunidades para los grupos más vulnerables. En este sentido, se consideró que para lograrlo se debían tener en cuenta los siguientes enfoques, dentro de cada una 
de las líneas estratégicas que posteriormente se delimitaron.

En primer lugar, se consideró que la generación de empleo en el municipio de Puerto Boyacá debía ir acompañada del desarrollo económico local territorial. Se trató de un proceso que buscó que el gobierno local conformara alianzas con otros actores estratégicos, con el fin de incentivar el crecimiento económico, a través de la construcción de un entorno competitivo que permitiera el fortalecimiento de las empresas, condiciones de trabajo digno y creación de nuevos empleos, que le apuntaran a la disminución de condiciones de pobreza. Dicho proceso implicó la conexión de políticas de desarrollo local, departamental y nacional en un marco integrador, que en este caso sería la Política Pública de Empleo (DEmuca, 2009).

De esta manera, se partió de comprender que para que haya desarrollo económico local se deben promover tres tipos de iniciativas: 1) las locales de empleo, 2) la promoción empresarial y, 3) aquellas orientadas a atraer nuevas inversiones económicas, mediante una estrategia de marketing del territorio. Asimismo, se tuvo en cuenta la importancia de crear entornos territoriales que facilitaran la incorporación de innovaciones productivas, nuevas empresas y empleos. Es por ello que se articuló la construcción de la política pública de empleo con el enfoque de desarrollo económico local territorial (Albuquerque, s. f.).

Al respecto, Mozas y Bernal (2006) señalan que las políticas de desarrollo han pasado de ser centralizadas a descentralizadas, en las que los actores del territorio tienen incidencia; además, son diseñadas con el fin satisfacer la demanda de los ciudadanos y empresarios, a partir de fortalecer el desarrollo de los territorios. Desde esta perspectiva se considera que "la nueva política tiene un enfoque territorial y entiende que la historia productiva, los recursos locales, las características tecnológicas e institucionales del contexto o el entorno condicionan el proceso de crecimiento" (p. 133).

Teniendo en cuenta la importancia de articulación del Desarrollo Económico Local Territorial con las políticas nacionales, el segundo enfoque para la Política Pública fue el de trabajo decente. Para ello, el Plan Nacional de Desarrollo (PND) 2014-2018, en el artículo 75, estableció la Política Nacional de Trabajo Decente con el fin de "promover la generación de empleo, la formalización laboral y la protección de los trabajadores de los sectores públicos y privados". Asimismo, señala que "las entidades territoriales formularán políticas de trabajo decente en sus planes de desarrollo, en concordancia con los lineamientos que expida el Ministerio del Trabajo".

En el marco de lo dispuesto en el PND 2014-2018, el Ministerio de Trabajo estableció cuatro componentes que deben tener este tipo de políticas, con base en los criterios establecidos por la Organización Internacional del Trabajo (оIт):

- Oportunidades de ingreso y empleo: dentro del PND se estableció que la creación de empleos se fundamenta en el Mecanismo de Protección al Cesante, estipulado en la Ley 1636 del 18 de junio de 2013, con el fin "garantizar la protección social de los trabajadores en caso de quedar desempleados, manteniendo el acceso a la salud, el ahorro a pensiones, su subsidio 
familiar y el acceso a servicios de intermediación y capacitación laboral". Esto mediante los siguientes mecanismos: 1) el Servicio Público de Empleo (sPe), 2) el Fondo de Solidaridad de Fomento al Empleo y Protección al Cesante (FOSFEC) y 3) las cuentas de cesantías.

- Derechos de los trabajadores: en el PND se adoptó la Política Nacional de Inspección, Vigilancia y Control orientada a "contribuir al fortalecimiento de las relaciones laborales, el respecto de derecho de asociación, el aumento en la afiliación y la protección al Sistema de Seguridad Social”.

- Protección social: a partir del establecimiento de políticas para fortalecer el Sistema de Protección y Seguridad Social, el cual garantizaría que los trabajadores pudieran elegir libremente las entidades a las que deseen estar afiliados.

- Diálogo social: fomentado a través de la Comisión Nacional de Concertación de Políticas Salariales y Laborales (CNCPSL).

Con el objetivo de que todas las personas tengan las mismas oportunidades de acceder a un empleo, la PPGE de Puerto Boyacá también se fundamentó en el enfoque diferencial. Esto con el fin de generar igualdad de oportunidades a las personas que han sido excluidas en razón de su etnia, sexo, ciclo vital, discapacidad, víctimas del conflicto armado, entre otras. De esta manera, se buscaba proteger la condición de ser distinto y diferente sin perder la capacidad de disfrutar las demás opciones humanas. En síntesis, el enfoque diferencial se entendió como:
El conjunto de acciones, que al dar trato diferenciado a algunos grupos poblacionales contribuye a reducir la brecha existente entre los diferentes segmentos de la población y garantiza la superación de la exclusión social, la marginalidad política, la desigualdad económica, la condición especial de vulnerabilidad y el riesgo de estas poblaciones ante el conflicto armado, lo que genera igualdad en el acceso a las oportunidades sociales (Ministerio del Interior, s. f., p. 4).

De esta manera, se consideró que los enfoques de desarrollo local territorial, trabajo decente y diferencial, son complementarios y esenciales para la generación de condiciones de bienestar de un territorio. Esto debido a que permitieron visionar el crecimiento económico de un territorio, teniendo en cuenta, las garantías laborales de los trabajadores, en igualdad de oportunidades.

\section{METODOLOGÍA DEL PROCESO DE CONSTRUCCIÓN DE LA FORMULACIÓN DE LA POLÍTICA PÚBLICA DE GENERACIÓN DE EMPLEO}

La política pública de generación de empleo de Puerto Boyacá se construyó de manera participativa, con el fin de contribuir a solucionar las problemáticas que obstaculizan el desarrollo local, que permitan crear empleo, $y$, por ende, mayores oportunidades para la población del municipio. Esto se realizó con base en la triangulación de información cualitativa y cuantitativa.

Como señala Sampieri (2014), el análisis cualitativo se puede entender "como un conjunto de prácticas interpretativas que al 
mundo 'visible' lo transforman y lo convierten en una serie de representaciones en forma de observaciones, anotaciones, [...] y documentos" (p. 9), a partir de lo cual, el investigador genera creencias propias sobre el fenómeno. El elemento clave para la recolección de datos es el investigador pues es quien, mediante diversos instrumentos, recolecta los datos y posteriormente los analiza. De esta manera, se constituye en el medio para recopilar información de diferente tipo: lenguaje escrito, verbal y no verbal, conductas e imágenes.

Así, el principal medio que se utilizó para la recolección de información fueron los talleres, los cuales se entienden como una forma de enseñar y aprender mediante la realización de actividades pedagógicas; es una metodología participativa en la que se aprende a través una tarea conjunta (Nolasco, s. f). A partir de ello se llevaron a cabo las siguientes actividades:

- Grupo focal con el Concejo Municipal, que contó con la participación de 16 dignatarios, y se realizó con el fin de conocer su percepción sobre la situación de empleo en el municipio (CER, 9 de septiembre de 2016).

- Dos talleres de priorización de actividades productivas, con el fin de identificar las potencialidades endógenas para el desarrollo territorial, contó en total con la participación de 69 personas, entre las cuales se encontraban empresarios tanto del sector urbano como rural (CER, $27 \mathrm{de}$ octubre y 10 de noviembre de 2016).

- Un taller de identificación de problemáticas, que fue replicado en la cabecera municipal, en el corregimiento de Puerto
Serviez y en las veredas Palagua y Muelle Velásquez; se contó con la participación de 150 personas en total. La participación de líderes de juntas de acción comunal (JAC), de organizaciones sociales y de la comunidad en general fue significativa (CER, 30 de noviembre, 1, 2 y 3 de diciembre de 2016).

- Dos talleres "Ruta de transformación" con el fin de identificar las líneas estratégicas para la superación de las problemáticas identificadas, el cual se realizó en la cabecera municipal y contó con la participación de 115 personas líderes de organizaciones sociales, asociaciones de desempleados, JAC provenientes tanto de la cabecera municipal como de los corregimientos y las veredas aledańas (CER, 18 y 25 de enero de 2017).

- Un taller de validación y retroalimentación preliminar de la PPGE, con el fin de realizar los ajustes pertinentes; contó con la participación de 42 personas, en su mayoría líderes de organizaciones sociales (CER, 01 de febrero de 2017).

- Socialización y entrega del documento de la PPGE, con la participación de 116 personas; se desataca la participación de los funcionarios de la Alcaldía Municipal, empresarios, líderes de organizaciones sociales y comunidad en general (CER, 18 de mayo de 2017).

- Observación participante y análisis cualitativo de la información recolectada, que permitió realizar una interpretación y comprensión hermenéutica de la situación socioeconómica del municipio. 
En cuanto al enfoque de análisis cuantitativo, Sampieri (2014) señala que se utiliza la lógica de razonamiento deductivo, por medio del cual se busca describir tendencias y patrones, evaluar variaciones, identificar diferencias y medir resultados, entre otros. A partir de ello se utilizaron los siguientes métodos:

- Se recolectó información secundaria en la que se recopilaron datos geográficos sobre el equipamiento municipal (servicios públicos, sistemas de comunicación e interconexión), situación sociocultural, aspectos económicos y productivos, y sobre el sistema político administrativo del municipio de Puerto Boyacá, con base en fuentes de información oficiales como el Departamento Administrativo Nacional de Estadística (DANE), el Sisben, el Ministerio de Educación entre otros.

- Se realizó un análisis estructural de las problemáticas identificadas con el software MicMac, a partir del cual se identificaron 40 factores de cambio; posteriormente se priorizaron nueve factores de cambio, los cuales fueron insumos para la elaboración de las líneas estratégicas.

Construir una política pública desde las comunidades de base, y las premisas de articulación y consenso, con y entre los actores gubernamentales-institucionales del sector privado y el sector comunitario implicó recorrer caminos diferentes a como ordinariamente se habían abordado estos temas. Como señala Albuquerque (1997, p. 323), "para que el proceso de de- sarrollo local genere una dinámica de cambio, es preciso que sea participativo y concertado".

En este sentido, se generó una dinámica constructivista que buscaba que los actores sociales (líderes de JAC y de organizaciones sociales), económicos (empresarios, gremios económicos, Cámara de Comercio de La Dorada) y políticos (alcalde municipal y secretarios de despacho) participaran, no como sujetos receptores de información, sino como cocreadores y críticos activos de la estructura teórica y práctica de los conceptos y las herramientas por trabajar, y que se caracterizan por tener un saber particular. En esa medida, la elaboración de la PPGE de Puerto Boyacá se nutrió con la experiencia individual y colectiva de los participantes, los cuales fueron insumos para su construcción.

A partir de ello es importante señalar que las rutas de construcción se basaron en procesos de diálogo y escucha activa. En consecuencia, se crearon propuestas pedagógicas y didácticas que permitieron que los actores participantes lograran consensos y acuerdos alrededor de su ejercicio de derechos y capacidad de agencia de su propio desarrollo. Asimismo, se facilitaron ambientes en los que los actores pudieron sentirse seguros y sin ningún tipo de recriminación.

A continuación se describen los momentos en los que se construyó de manera participativa la formulación de la PPGE de Puerto Boyacá, en la que se describen los resultados obtenidos en cada uno de ellos, con base en los métodos e instrumentos de recolección de información señalados. 


\section{Diagnóstico socioeconómico participativo}

Parte del éxito del proceso estuvo en conocer las condiciones actuales del territorio frente al tema de empleabilidad y productividad. Teniendo en cuenta que algunos entes territoriales carecen de información actualizada de su realidad socioeconómica, se llevaron a cabo acciones de campo que permitieron realizar el análisis socioeconómico a partir de la triangulación de información primaria (talleres, observación participante) y secundaria (datos estadísticos, fuentes nacionales).

De esta manera, se recolectó información secundaria con la que se buscó conocer la realidad económica y de gestión pública del municipio, que diera cuenta de las potencialidades del territorio y de los sectores que requerían ser fortalecidos para su desarrollo en el corto, mediano y largo plazo, frente a variables como: 1) geografía, 2) demografía, 3) etnografía, 4) gestión pública, 5) educación, 6) población víctima, 7) servicios públicos, 8) seguridad social, 9) uso del suelo, 10) principales actividades económicas, 11) cadenas productivas y mercado laboral.

Para recolectar información primaria se realizó un taller de identificación de problemáticas alrededor del empleo; este se replicó en la cabecera municipal, en el corregimiento de Puerto Serviez y en las veredas de Muelle Velásquez y Palagua, de manera que se lograra una participación representativa en la construcción de la política.

Fueron jornadas de trabajo que contaron con un permanente acompañamiento para lograr generar diálogos constructivos que permitieron obtener insumos para la elaboración de la política. Por ello, se crearon herramientas de manejo de emociones y protocolos de debate que, con una adecuada facilitación, brindaron un ambiente controlado, y con todo el sentido de libertad por parte de cada actor.

A partir del análisis de las problemáticas identificadas por los actores participantes se halló que estas se deben, en su mayoría, a externalidades negativas de la industria del petróleo, en especial por el deseo de la mayor parte de la población de acceder a un empleo formal, con prelación de la mano de obra local. A continuación se señalan cinco tópicos que integran los datos recogidos en el diagnóstico, así como la información obtenida en los talleres participativos de identificación de problemáticas.

\section{Círculo de dependencia del sector hidrocarburos}

Uno de los enfoques que contribuyeron a comprender la realidad económica y sociopolítica de Puerto Boyacá fue la llamada "maldición de los recursos naturales" o paradoja de la abundancia, si se tiene en cuenta que es un municipio rico en recursos naturales, pero con un bajo crecimiento económico. Según Morales (2012), esto implica preguntarse cómo las economías que están dotadas de una mayor riqueza natural son las de menor desarrollo, mientras que aquellas que cuentan con escasos recursos crecen a ritmos acelerados. $\mathrm{Al}$ respecto, Ross (1999) señala que esto se debe a un tipo de miopía en los sectores públicos y privados, en especial porque no se genera diversificación económica, y el crecimiento económico se basa en la búsqueda de beneficios en el corto plazo. 
Durante el periodo 2011-2013, el producto interno bruto (PIB) de Puerto Boyacá superó los 2.000 millones de pesos, alcanzando en 2012 el valor de 2.842 millones, el más alto en el periodo analizado. A pesar de ello, es necesario resaltar que la economía de este municipio depende en gran parte del sector minero, tal como se puede ver en la figura 2 . Más del $80 \%$ del piв municipal fue aportado por este sector, principalmente por la actividad petrolera (CER, 2016).

FIGURA 2. PIB PUERTO BOYACÁ POR SECTORES ECONÓMICOS, 2011-2013

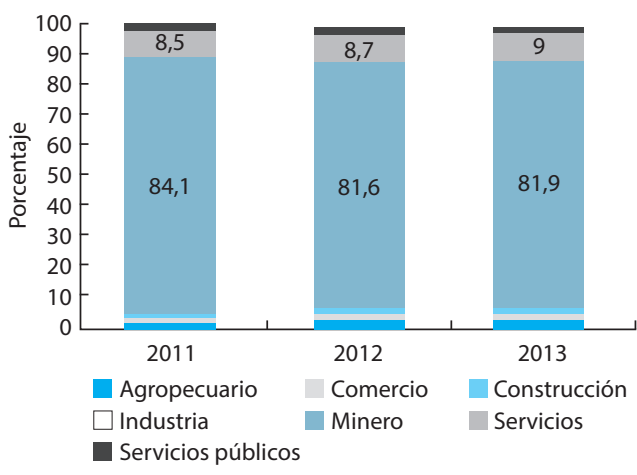

Fuente: DANE (2013), cálculos CER (2017).

Adicionalmente, los demás sectores económicos del municipio generaron un bajo aporte en relación con el PIB total. Es de resaltar, por ejemplo, que en el ámbito departamental el sector servicios fue el que generó mayor desarrollado e ingresos, mientras que para este territorio solo aportó cerca del $9 \%$ durante el trienio (CER, 2016).

Puerto Boyacá presenta una debilidad en el desarrollo rural, puesto que más del $80 \%$ de su población reside en el casco urbano, aun cuando el $97 \%$ de su territorio es rural. De otro lado, el $80 \%$ de las tierras se utilizan en desarrollo de ganadería extensiva, actividad que genera riqueza para pocos y limitadas opciones de generación de empleo, dado que no es intensivo en mano de obra, en vez de actividades agrícolas que pudieran generar una cantidad importante de empleos (CER, 2016).

Los datos recolectados y el diálogo con las comunidades durante los talleres participativos permitieron identificar que se ha generado un círculo de dependencia del sector de hidrocarburos que consiste en: 1) llegan las empresas de hidrocarburos a territorios con pocos recursos económicos; 2) los empleos generados por este sector son bien remunerados, incluyendo aquellos que implican mano de obra no calificada, y otorgan las prestaciones sociales; 3 ) las comunidades prefieren cambiar su actividad productiva tradicional por un empleo en el sector hidrocarburos, por lo cual comienzan a exigir la contratación de mano de obra local; 4) ante la creciente demanda de empleo, las instituciones técnicas y tecnológicas comienzan a ofrecer oportunidades de formación en este sector; 5) hay un gran número de personas formadas y no formadas con expectativas de acceder a un puesto de trabajo en el sector hidrocarburos; 6) la demanda de mano de obra en este sector no es suficiente, y es vulnerable a los precios internacionales de hidrocarburos; 7) la comunidad se siente insatisfecha por no tener las oportunidades laborales, de manera que se genera una competencia legal, y en ocasiones ilegal (compra de certificados de territorialidad, clientelismo, entre otros) para acceder al empleo; 8) se relegan las actividades productivas propias del territorio (agricultura, ganadería, turismo y demás), de manera que son cada vez más dependientes de las opor- 
tunidades que pueda ofrecer este sector (CER, 2016).

De esta manera se llega a un punto de difícil retorno, dado que el cambio de vocación productiva requiere que los actores del territorio estén dispuestos a poner en marcha procesos de emprendimiento que generen empleos formales y bien remunerados, que por lo general no implican cambios en el corto plazo. Como seńala Stiglitz uno de los mecanismos para la transformación social es facilitar un ambiente propicio para los negocios "que no solo traiga a los inversionistas extranjeros, sino que ofrezca un entorno agradable a los inversionistas nacionales" (2003, p. 34).

\section{Formalización laboral frente a oportunidades de empleo para todos}

La población en edad de trabajar (PET) se divide en población económicamente activa (PEA) y en inactivos. Dentro de esta primera subdivisión se encuentran todos aquellos que en la actualidad tienen un trabajo o están en búsqueda de uno. Por su parte, los inactivos hace referencia a aquellas personas que se dedican únicamente a estudiar, amas de casa, rentistas, jubilados, pensionados, personas con alguna discapacidad o invalidez permanente para trabajar, o aquellos que manifiestan no tener una actividad determinada. De esta manera, la PET de Puerto Boyacá en el 2016 constituía el 79,5\% de la población total, el $20,5 \%$ restante eran nińos menores de 12 años (CER, 2016) (figura 3).

\footnotetext{
1 Tasa de ocupación $=($ Ocupados/PET $) \times 100$.

2 Tasa de desempleo $=($ Desocupados/PEA $) \times 100$.
}

De otro lado, en Puerto Boyacá la población inactiva (23.272 personas) es mayor a la económicamente activa (18.409 personas), lo que mostró que en el municipio hay una gran cantidad de jóvenes estudiantes, amas de casa y pensionados o rentistas. A su vez, la tasa de ocupación $^{1}$ del municipio es del 34,8\%. Por su parte, la tasa de desempleo ${ }^{2}$ es del $21,2 \%$, un nivel bastante alto en comparación del nivel promedio de desempleo nacional que en el 2016 estaba alrededor del 9\% (CER, 2016b).

\section{FIGURA 3. MERCADO LABORAL DE PUERTO BOYACÁ, 2016}

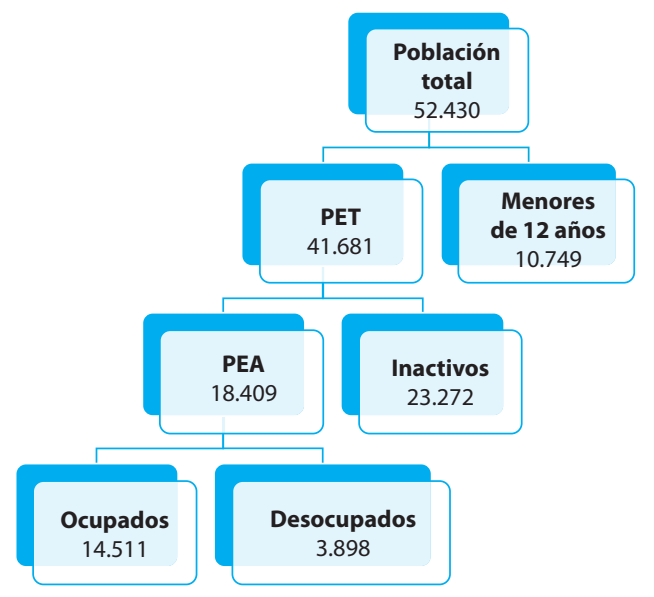

Fuente: Sisben (2016). Los datos de mercado laboral tomados del Sisben son aproximados, ya que no toman a la totalidad de la población; además, son recopilados en diversos momentos del año, por lo cual el mecanismo más cercano sería contar con información de una encuesta de hogares.

Ante las pocas oportunidades laborales y el deseo de obtener un puesto de trabajo en el sector hidrocarburos, las comunidades optaron por usar estrategias de rotación del empleo, por 
medio de las JAC, buscando que la mayor parte de los miembros de la comunidad tuvieran una oportunidad laboral, así fuera por tan solo unos meses. De esta manera, las JAC se encargaban de recibir las hojas de vida, así como de estipular el tiempo en que las personas debían ser contratadas, buscando que la mayor parte de los miembros de la comunidad pudieran acceder a un puesto de trabajo. Por ejemplo, un cargo que requería el sector para una persona durante un año, podría ser distribuido en cuatro personas, quienes trabajarían cada una por tres meses (CER, 2016).

A partir de ello, el Gobierno nacional expidió el Decreto 1668 de 2016, como medida para garantizar la igualdad de oportunidades en la contratación de mano de obra local, en municipios donde se desarrollen proyectos de exploración y producción de hidrocarburos. De esta manera, se definió que el proceso de priorización de la demanda laboral local se realizaría mediante los prestadores autorizados del servicio público de empleo, e inhabilitó a las JAC para llevar a cabo esta función.

Esta acción generó resistencia por algunos miembros de la población, en especial porque impidió la rotación de empleo, como se venía haciendo; esto mostró que era más importante la posibilidad de trabajar unos meses en la industria, que la estabilidad laboral. A partir de ello, en los talleres participativos se encontró que una de las inconformidades de la comunidad era el hecho de que algunos habitantes del municipio mantenían su puesto de trabajo, y limitaban la posibilidad de que otras personas pudieran acceder al empleo. Esto denotó una cultura de dependencia en el sector hidrocarburos que podría afectar la generación de una dinámica laboral orientada a la estabilidad, formalización y diversificación de los puestos de trabajo (CER, 2016).

Es importante resaltar que la reciente crisis económica que generó la caída de los precios del petróleo en el 2015, sobre todo en los municipios de mayor explotación mineroenergética, podría aumentar las posibilidades de que la población inactiva pase a demandar empleo, debido a la necesidad de conseguir ingresos para satisfacer necesidades básicas. A partir de ello, se tuvo en cuenta que la presencia de este tipo de población en cualquier momento podría llegar a engrosar las cifras de personas desocupadas, aumentando la percepción de crisis, y, por ende, el descontento y malestar social.

Al respecto, Schuldt y Acosta (2006), en su artículo "Petróleo, rentismo y subdesarrollo ¿una maldición sin solución?” hacen referencia a que una de las patologías que impide el crecimiento económico de los territorios ricos en petróleo es la tendencia a generar desempleo, subempleo y pobreza, consolidando la desigual en la distribución de los ingresos y los activos.

\section{Desinformación y pocos canales de comunicación formales}

De otro lado, durante el Taller de Identificación de Problemáticas (2016), se halló que parte de la población de Puerto Boyacá no tiene conocimientos claros acerca de las normas que regulan el acceso al empleo en el sector hidrocarburos, en especial con la implementación del Decreto 1668 de 2016, esto se debe a: 1) falta de canales de comunicación formales; en ese sentido, se encontró que se confía más en la información que otorgan los líderes sociales -los cuales son las personas 
con las que están en permanente contacto-, que aquella suministrada por la Alcaldía Municipal o las empresas. 2) La desinformación se da cuando no se transmite con claridad a la comunidad cuáles son los procesos legales para acceder al empleo, generando tergiversación de la información y conjeturas al respecto. Entre los aspectos que permiten evidenciar lo anterior están:

- Delimitación de criterios para Certificado de Territorialidad: miembros de la comunidad, con el ánimo de proteger la mano de obra local, propusieron la entrega del Certificado solo a personas con cédula del municipio de Puerto Boyacá; además de que esto no es lo estipulado por la ley, dejaría por fuera personas que no nacieron en el municipio pero llevan muchos años viviendo en él.

- Confusión frente a las funciones del servicio público de empleo: la comunidad todavía se refiere a las bolsas de empleo y las JAC como si fueran las encargadas de la organización del empleo.

- Dudas frente a lo que implica la ley del primer empleo. Se tiene la percepción de que la ley es para la población en general, y no para los jóvenes recién graduados. De esta manera, son recurrentes los señalamientos frente a que no se les dan oportunidades laborales a aquellas personas con formación, pero sin experiencia. Esto se presenta porque muchos de ellos, sin importar la edad, se capacitaron para obtener un empleo en la industria y posteriormente se encontraron con las dificultades de la experiencia.
Al respecto, Patricia Suárez (2011), en su texto El Servicio Público de Empleo en España: ensayos desde una perspectiva regional, dice que la intermediación laboral mejora en la medi$\mathrm{da}$ en que los trabajadores tienen acceso a la información sobre las vacantes disponibles. Sin embargo, considera que se pueden generar las siguientes asimetrías informativas: 1) la información de las vacantes varía según el mecanismo que se utiliza, por ejemplo, diferencias en los datos otorgados en la Oficina de Empleo con aquella a la que se puede acceder en la plataforma virtual; 2) selección adversa, la cual parte de la concepción de las empresas sobre que en el SPE se inscriben los peores trabajadores y a la inversa. De esta manera, se infiere que es frecuente que el uso de este tipo de plataformas públicas genere asimetrías de información, y por ende desconfianza.

\section{Desconfianza de las comunidades en las instituciones públicas y privadas}

Lo que sucede en Puerto Boyacá no es lejano a lo que Rosanvallon (2007) denomina la "sociedad de la desconfianza", la cual parte del distanciamiento entre los individuos; en general, es una falta de confianza en el prójimo y hacia los gobernantes los cuales, según el autor, están correlacionados. De esta manera señala que hay dos tipos de desconfianza: la liberal y la democrática. La primera está orientada a la prevención de la acumulación de poderes; la segunda busca velar por que los que están en el poder lleven a cabo sus compromisos, así como los medios que se utilizan para cumplir tales fines.

Lo anterior se ve plasmado en tres modalidades principales: 1) los poderes de control, 
que en el caso de Puerto Boyacá se ven reflejados en la percepción de que la Alcaldía Municipal y el spe le dan prioridad a beneficios a terceros; 2) las formas de obstrucción, que se materializan en los paros y las protestas; y 3) la puesta a prueba a través del juicio moral, que se evidencia en las asunciones de que la Alcaldía y el SPE manipulan intencionalmente el otorgar o no, tanto el certificado laboral como las vacantes laborales.

Con base en ello, se halló que los actores sociales de Puerto Boyacá desconfían del proceso de selección de personal que se da a través de la plataforma del SPE administrada en el municipio, y también del proceso de entrega del certificado de territorialidad porque consideran que: 1) se le da prioridad a la mano de obra foránea; 2) las personas encargadas de estos trámites manipulan los procedimientos para beneficiar a sus amigos o personas cercanas; 3 ) las personas foráneas que tienen mayor poder adquisitivo compran el certificado de territorialidad; 4) existe la percepción de que las oportunidades laborales deben ser para toda la población, y piensan que quienes han logrado obtener una oportunidad laboral la han hecho de manera injusta, sea porque han tenido un vínculo familiar o han comprado un cupo; de esta manera se desconoce que las personas que han adquirido un empleo lo han hecho por sus propias competencias; 5) se asume que cuando una persona obtiene posibilidades de manera ilegal es responsabilidad de la empresa o la Alcaldía Municipal, mas no de problemas propios de una cultura del atajo y la ilegalidad (Taller Diagnóstico Participativo, 2016).

\section{Brechas sociales}

Según Silva (2005), la búsqueda por generar condiciones de igualdad es uno de los objetivos estratégicos del desarrollo, lo que requiere, por un lado, realizar cambios estructurales, $y$, por otro lado, usar la política como instrumento. El primero implica la transformación y diversificación de la estructura productiva a través de la incorporación de innovación tecnológica, del fortalecimiento del conocimiento, la inclusión de los sectores informales a los procesos de desarrollo y el desarrollo de capacidades de las nuevas generaciones. El segundo señala que para que esto se dé se requiere de políticas económicas y productivas, así como del fortalecimiento del diálogo social.

$\mathrm{Al}$ respecto, es importante señalar que Puerto Boyacá tiene grandes retos respecto a la generación de cambios que contribuyan a la generación de condiciones de igualdad, puesto que no cuenta con la infraestructura y los equipamientos (instituciones educativas, puestos de salud), acceso a la educación, acceso a tecnologías de información que le permitan ser competitivo con otros municipios y regiones del país (CER, 2016). Solo por señalar algunas cifras, el 16\% (8.800 personas) de la población se considera como analfabeta ${ }^{3}$; de estos, el $23 \%$ reside en el ámbito rural (рвот, 2012) (figura 4).

\footnotetext{
3 Analfabeta: persona que no sabe leer ni escribir. Para el documento diagnóstico se realizó el cálculo con población mayor de 5 años de edad.
} 
FIGURA 4. ÚLTIMO NIVEL EDUCATIVO ALCANZADO POR LA POBLACIÓN DE PUERTO BOYACÁ

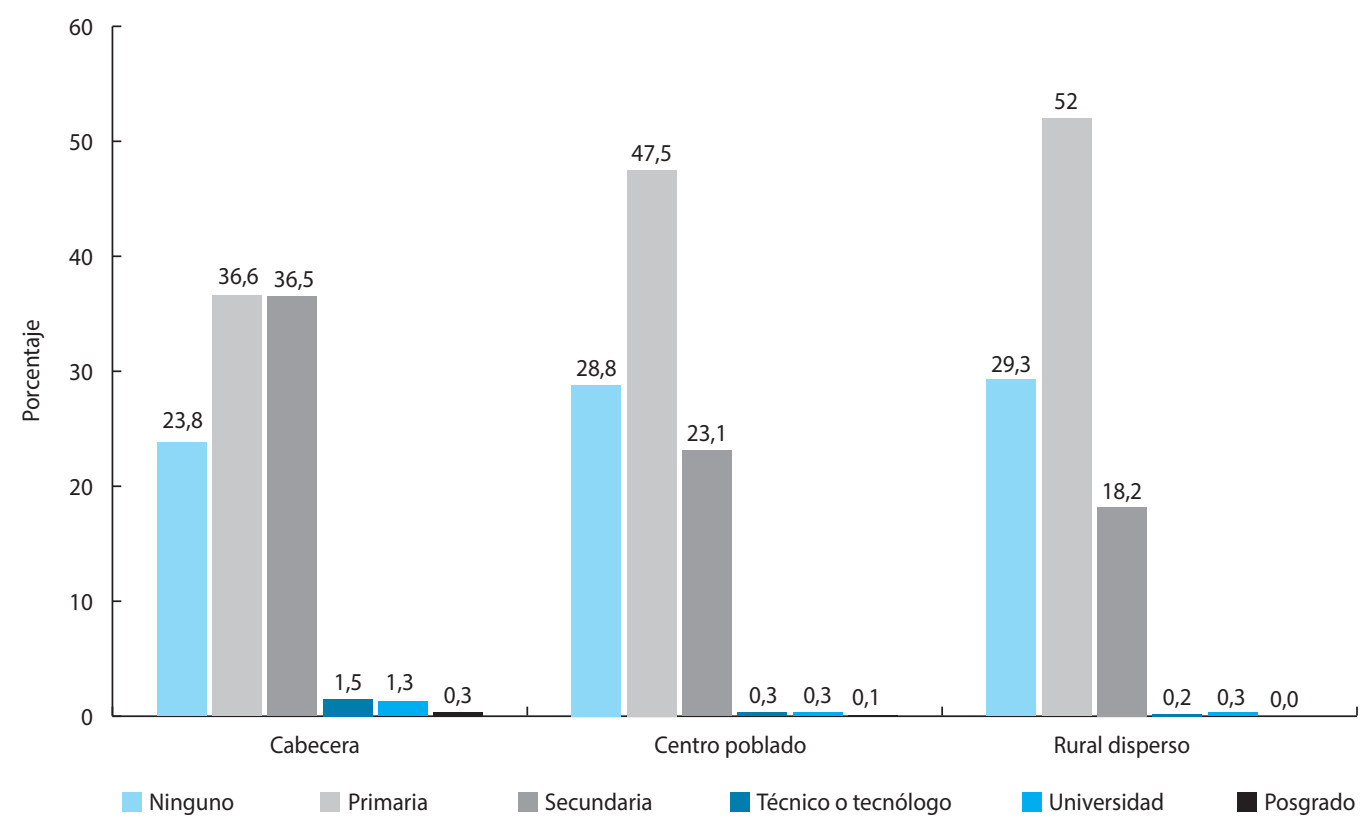

Fuente: Sisben, 2016. Cálculos CER (2017).

De esta manera, para la implementación de la política pública de generación de empleo se identificaron dos aspectos importantes desde el punto de vista educativo: en primer lugar, facilitar la llegada de diferentes instituciones educativas y de formación profesional con el fin de aumentar la cobertura y la variedad de los programas ofrecidos en el municipio, esto buscando ampliar el menú de opciones para los futuros estudiantes y evitar así la fuga de personal educado hacia otros lugares del país. Segundo, iniciar acciones de mejora con el fin de reducir los niveles de analfabetismo del municipio; si bien este fenómeno no depende del área en donde se encuentra ubicada la persona, puede ser que se deba a un proceso cultural o familiar.

\section{Priorización de actividades productivas}

Uno de los aspectos claves para el desarrollo económico local es la generación de ventajas competitivas en torno a propiciar la conformación de pequeñas y medianas empresas que busquen generar aglomeraciones productivas (cluster) y economías de escala, que les permitan competir con empresas en el mercado global. Esto implica la transformación de los sistemas productivos locales. En este sentido, "se plantea que el desarrollo local y regional es un proceso eminentemente endógeno que procura aprovechar sus potencialidades propias -naturales, humanas, institucionales y organizacionales- para transformar los sistemas productivos locales con el propósito de mejorar la calidad de vida de la población" (Silva, 2005). 
Con base en esto se realizaron dos talleres cuyo fin era identificar las potencialidades productivas del municipio, que se caracterizaran por ser las más viables y de mayor impacto, lo cual se llevó a cabo a través de un análisis valorativo fundamentado en una escala de medición cuantificable, que permitió establecer un ranking. Esto se implementó con base en la propuesta metodológica "Identificación de actividades económicas y productivas de mayor desarrollo en el ámbito municipal", del Programa de las Naciones Unidas para el Desarrollo (pNUD).

Con la variable impacto se buscó valorar aquellas actividades económicas generadoras de empleo, valor y riqueza en el largo plazo, lo cual se debe ver reflejado en que la rentabilidad sea mayor que el capital invertido (PNUD, s. f.). Con la variable viabilidad se buscó valorar si el desarrollo de la actividad económica cuenta con: 1) la voluntad política, 2) capacidad organizacional, 3) canales de comercialización, 4) infraestructura y 5) criterios de sostenibilidad, que generen las condiciones ideales para su posicionamiento en el mercado (PNUD, s. f.).

Por consiguiente, la valoración de las actividades económicas se hizo través de una metodología cualitativa y cuantitativa. La medición del impacto y la viabilidad se realizó mediante siete y seis preguntas respectivamente, a partir de las cuales se asignó una valoración cuantitativa tomando como referencia una escala de 0 a 1300. Como resultado de la ponderación de puntajes obtenidos en cada mesa de trabajo se obtuvo el siguiente ranking de actividades productivas (tabla 1).

Posteriormente las nueve actividades productivas identificadas se agruparon en seis: 1) pancoger (cacao, plátano, yuca y maíz), 2) piscicultura y pesca artesanal, 3) frutales, 4) turismo ecológico, 5) transporte fluvial y terrestre y 6) ganadería. En el segundo taller se buscó identificar las problemáticas alrededor de la actividad que obstaculizan que esta sea viable y tenga un impacto positivo en la economía. Es decir, las posibles causas de fondo

TABLA 1. VALORACIÓN Y RANKING DE ACTIVIDADES PRODUCTIVAS

\begin{tabular}{|r|l|}
\hline \multicolumn{1}{|c|}{ Escala } \\
\hline \multicolumn{2}{|c|}{ RANKING } \\
\hline 1300 & Muy alto \\
\hline 975 & Alto \\
\hline 650 & Medio \\
\hline 325 & Bajo \\
\hline 0 & Nulo \\
\hline
\end{tabular}

\begin{tabular}{|c|l|c|c|c|}
\hline $\mathbf{N}^{\circ}$ & \multicolumn{1}{|c|}{ Actividad económica } & Impacto & Viabilidad & \\
\hline 1 & Turismo ecológico & & & 850 \\
\hline 2 & Piscicultura y pesca artesanal & - & & 888 \\
\hline 3 & Frutales & - & & 854 \\
\hline 4 & Ganadería doble propósito & - & & 796 \\
\hline 5 & Actividad agroforestal & - & & 604 \\
\hline 6 & Plátano y yuca & - & & 850 \\
\hline 7 & Maíz & - & & 854 \\
\hline 8 & Transporte fluvial y terrestre & 0 & & 821 \\
\hline 9 & Cacao & & & 933 \\
\hline
\end{tabular}

Fuente: CER (2016) 
que impiden el crecimiento de la actividad económica desde los recursos económicos, el entorno, capital humano, políticas gubernamentales, entre otras.

Esta actividad se desarrolló mediante la metodología de Metaplan ${ }^{4}$, en la que se le pidió a los participantes que escribieran de manera sintética, en una ficha, las problemáticas que desde su conocimiento y experiencia impiden el desarrollo de la actividad económica que realizan. Finalmente, se concretaron siete problemáticas por cada actividad

Vía matriz de Véster se valoró la relación causa-efecto de los diversos problemas, a fin de medir la motricidad y dependencia de estos. Esta metodología permitió: 1) analizar las relaciones entre los elementos de un sistema, 2) concentrarse en sus variables más significativas.

Siguiendo con la metodología, los resultados de la votación se ingresaron en un plano cartesiano con el fin de identificar y priorizar las problemáticas en función de: problemas indiferentes, pasivos, activos y críticos; en su orden constituyen los de menor y mayor priorización.

Los críticos se definen como problemas de gran causalidad y estos son de vital importancia, por lo que las alternativas de solución se plantearon alrededor de ellos. Las problemáticas críticas comunes en las seis actividades productivas fueron: 1) no hay apoyo de los gobiernos nacional y local; 2) falta de tierras e infraestructura, y 3 ) ausencia de encadenamientos productivos. Teniendo claro las pro- blemáticas por cada unas de las actividades económicas y su relación de causalidad, en las mesas de trabajo se procedió a identificar y ordenar alternativas de solución para superarlas.

\section{RUTAS DE TRANSFORMACIÓN. DEL PROBLEMA A LAS LÍNEAS DE ACCIÓN}

Como señala Stiglitz (2003), uno de los mayores retos para el desarrollo es la transformación social, a través de un ambiente propicio para los negocios. Al respecto la CEPAL (2017), en el texto "Desarrollo económico local y competitividad territorial", muestra que en la nueva economía global solo pueden competir aquellos territorios que tienen la capacidad de aprender y adaptarse a la dinámica productiva mundial. A partir de ello señalan: "esto implica que los territorio subnacionales necesitan desarrollar sus respectivas habilidades y ventajas, o su capacidad de construirlas, para especializarse en áreas o sectores que tengan posibilidades de inserción internacional" (Silva, 2005, p. 82).

De esta manera, plantea que hay dos lógicas de división de los territorios: la vertical y la horizontal. La primera corresponde a las empresas transnacionales que pueden privilegiar los procesos de selectividad territorial. La segunda está relacionada con la idea de construcción social de los territorios que se caractericen por ser innovadores y competitivos. Para ello, se requiere propiciar espacios de encuentro entre los actores - públicos, sociales

\footnotetext{
4 Técnica de visualización fundamentada en la utilización de tarjetas de colores que facilitó la participación de los involucrados en el taller.
} 
y privados- a fin de modificar el aparato productivo (Silva, 2005).

Con base en esto se realizó un taller para generar un espacio de construcción social del territorio que partiera de identificar los aprendizajes obtenidos hasta el momento, en el proceso de desarrollo económico local para, a partir de ello, definir los cambios que se requieren para generar la transformación del sistema productivo, y, por ende, aumentar la generación de empleo.

Una de las herramientas construidas para el logro de tal efecto fueron los núcleos de análisis propositivos (NAP), a partir de los cuales se construyeron cinco casos que motivaron la reflexión y el análisis con respecto a las diferentes problemáticas identificadas en el taller, que se caracterizan por obstaculizar el desarrollo del territorio; estos se llevaron a cabo sobre los siguientes temas: 1) desarrollo de una estrategia de emprendimiento, 2) fortalecimiento de procesos productivos, 3) normatividad labo- ral, 4) formación en el territorio, 5) gestión y articulación institucional.

Los casos se narraron en un lenguaje no técnico, pero con elementos de profundidad para realizar un análisis efectivo de la interpretación por parte de todos los actores, pero a la vez, en un enfoque propositivo, buscando la estructuración de "rutas de transformación" con las cuales se lograra cambiar la situación problema en oportunidad de mejora para el entorno, traducido en su momento como política pública (figura 5).

En el análisis de caso se llevó a cabo un ejercicio de cambio de roles, lo que permitió no solo que se realizara el ejercicio controlado emocionalmente, sino con un alto grado de toma de conciencia por parte de los actores del rol que cada uno desempeńa dentro del proceso de generación de empleo. En general, con esto se buscó iniciar un proceso de resignificación mutua y reconocimiento entre los actores en forma no violenta.

FIGURA 5. RUTA DE TRANSFORMACIÓN

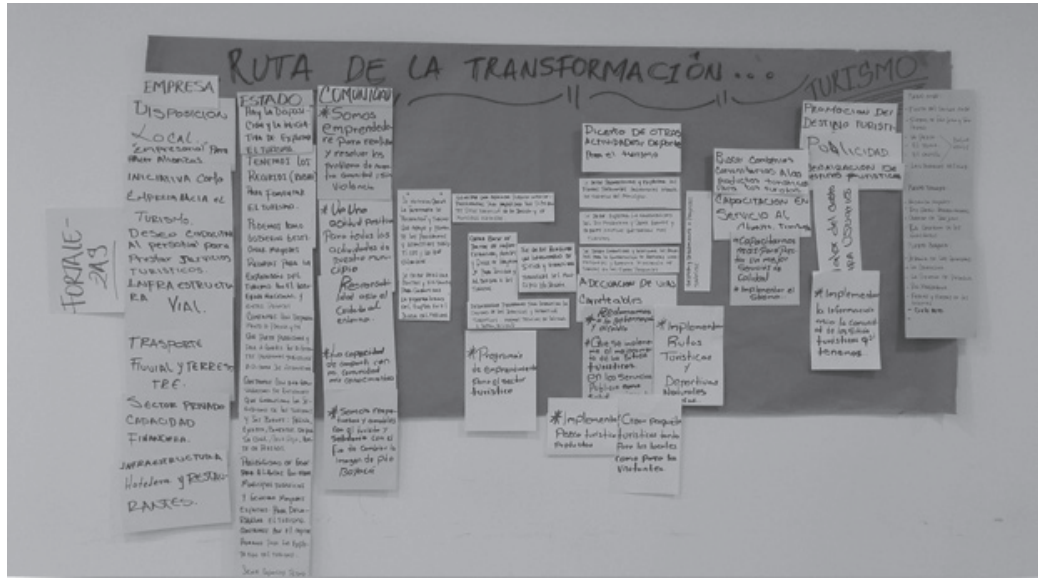

Fuente: Taller de Ruta de Transformación, 25 de enero del 2017, Puerto Boyacá. 
El adecuado análisis de los NAP permitió establecer relaciones entre temas, actividades y estrategias, y se obtuvo una priorización y organización pertinente de los elementos que la política pública debía abordar, además de establecer los insumos de implementación, evaluación y seguimiento de esta.

Con la información recolectada en los talleres, y el diagnóstico, se identificaron 40 factores de cambio, es decir, aquellos elementos significativos, susceptibles de generar transformaciones en el territorio. Posteriormente, se realizó un análisis estructural el cual es "un método sistemático, en forma matricial, de análisis de las relaciones entre variables constitutivas del sistema estudiado y las de su entorno explicativo" (Godet y Durance, 2009, p. 54). Para ello se utilizó el software Matriz de Impactos Cruzados, Multiplicación Aplicada a una Clasificación (MICMAC), el cual permite evaluar qué tanto incide una variable sobre otra, en una valoración de 0 a 3, con el fin de identificar las variables más influyentes y menos dependientes de las demás. A partir de ello se priorizaron los siguientes nueve factores de cambio (CER, 2016b) ${ }^{5}$ :

- Tejido económico: maneras o formas como se comportan los diversos actores del territorio entre sí, en especial con relación a sus acciones de generación de ingresos y aprovechamiento de los recursos del territorio para el bienestar económico de todos los actores.
- Diversificación económica: capacidades y potencialidades de sectores económicos (agropecuario, agroindustrial y turismo) en el territorio, los cuales generan o fortalecen actividades de lucro y generación de ingresos para los diferentes actores; asimismo, nuevas oportunidades de negocio, sostenibles en los mercados actuales y emergentes.

- Proyección territorial: visión del territorio como generador de bienestar integral para sus habitantes con un interés en su proyección empresarial y de emprendimiento.

- Formación del talento humano: estado en que se encuentran los recursos educativos del territorio en beneficio de sus habitantes, en su acceso, oferta y pertinencia.

- Emprendimiento local: capacidades individuales y colectivas de los actores del territorio para generar nuevas formas de ingresos y aprovechamiento de los recursos, desarrollando factores de sostenibilidad en el mercado y políticas a corto, mediano y largo plazo.

- Normatividad laboral y empresarial: todo lo relacionado con la elaboración y el cumplimiento de normatividad y leyes en pro de una regulación adecuada de recursos y manejo laboral del territorio, previniendo y denunciando acciones ilícitas.

5 Es importante señalar que dichos factores se construyeron a partir de los aportes de los participantes a los talleres, así como de un análisis hermenéutico de la información recolectada. 
- Finanzas públicas: capacidad de buen manejo de ingresos e inversiones de dinero público en pro de generar bienestar a los actores del territorio.

- Capacidad de gestión institucional: capacidad de los actores gubernamentales y privados del territorio para garantizar gobernabilidad y liderazgo de sus políticas, en pro de cumplir con sus responsabilidades en la provisión de servicios a la ciudadanía, garantizando el uso eficiente de los recursos y trabajando articuladamente en el diseño y la ejecución de programas de inversión de interés regional.
- Sector hidrocarburos: manejo de recursos de explotación del petróleo en el territorio e identificación de prácticas que lleven a la no dependencia en del tejido económico presente en el territorio.

\section{Lineamientos estratégicos de la formulación de la PPGE}

A partir del análisis de la problemática, la identificación de los factores de cambio y los aprendizajes obtenidos de los actores representativos de los sectores públicos, privados, gremios económicos, asociaciones, instituciones educativas y comunidad en general, se construyeron

FIGURA 6. LÍNEAS DE LA POLÍTICA PÚBLICA DE EMPLEO

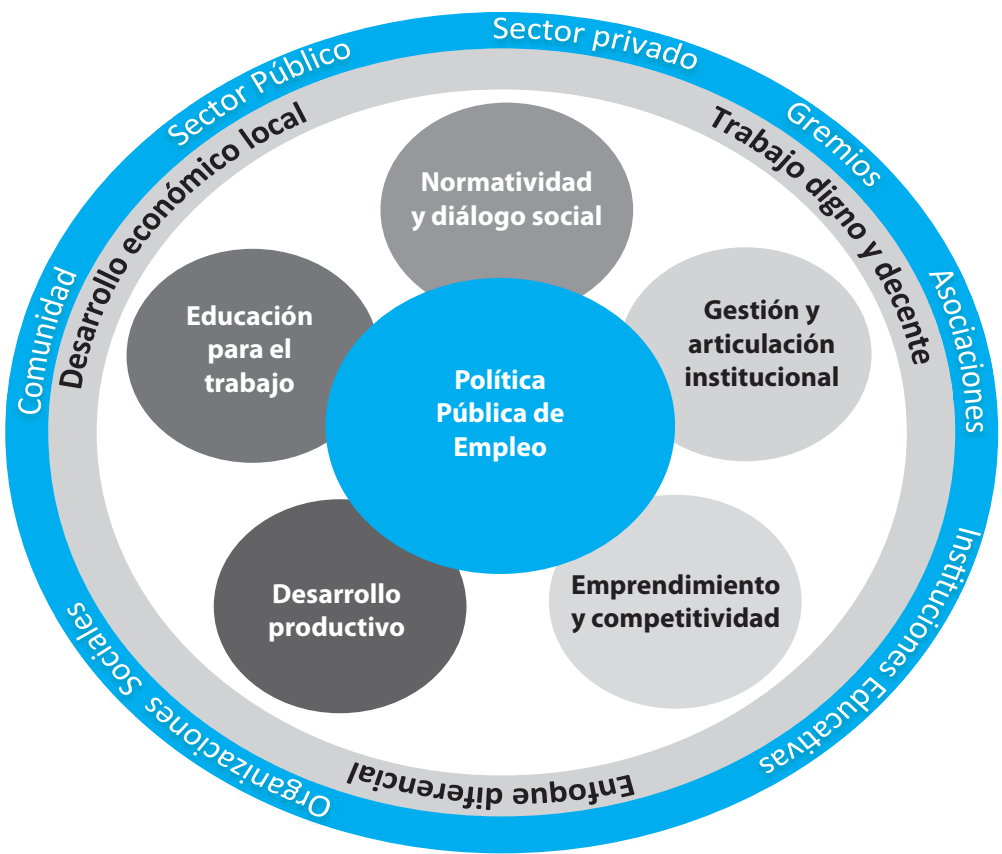

Fuente: CER (2017). 
los lineamientos que se consideran serán estratégicos para la transformación productiva del territorio, los cuales son: 1) fortalecimiento normativo y diálogo social, 2) educación para el trabajo, 3) gestión y articulación institucional, 4) desarrollo productivo, y 5) emprendimiento y competitividad (figura 6).

- Fortalecimiento normativo y diálogo social. Buscó establecer lineamientos y directrices que orientaran a los actores públicos, privados y sociales al cumplimiento de las normas, como medida para garantizar los derechos y deberes laborales, mediante escenarios que promuevan la conversación, la transparencia, la legitimidad y el trabajo decente.

- Educación para el trabajo. Se orientó en elaborar programas a fin de formar para el trabajo a la población, en articulación con los sectores público, privado y académico.

Gestión y articulación institucional. Se basó en diseñar e implementar una estrategia de promoción de articulación entre las instituciones públicas, las empresas y las organizaciones sociales, con el fin de aportar al desarrollo socioeconómico del municipio.

Desarrollo productivo. Se propuso con el fin de potencializar la productividad, aprovechando las vocaciones del territorio, con el fin de dinamizar la economía y ampliar las oportunidades para la generación de empleo.

Emprendimiento y competitividad. Se basó en mejorar la generación de ingresos y emprendimientos, a través del fortaleci- miento de los procesos de asociatividad, y la capacidad técnica y de gestión de financiación que facilitarán el desarrollo de iniciativas empresariales enfocadas en la vocación productiva.

Además, se revisó el marco jurídico colombiano frente a qué tanto protege los derechos laborales, cómo incentiva la generación de empleo, el emprendimiento y la formación para el trabajo. Con estos insumos se elaboraron los programas y las iniciativas de proyectos por cada una de las líneas. Estos últimos se priorizaron en: a) corto plazo, con base en los programas que pueden ser financiados por los planes de desarrollo nacional, departamental y local actuales; b) mediano plazo, aquellos que requieren la gestión de financiación en las diversas instituciones públicas y privadas; y c) largo plazo, aquellos que por sus características requieren de mayor inversión y cuyos impactos de implementación se verán reflejados en el futuro.

\section{CONCLUSIONES Y APRENDIZAJES DEL PROCESO DE CONSTRUCCIÓN DE LA FORMULACIÓN DE LA POLÍTICA PÚBLICA DE EMPLEO}

El proceso de construcción de las políticas públicas de generación de empleo fue un espacio de diálogo social, que permitió que los diferentes actores acordaran la proyección del desarrollo económico de sus municipios. Asimismo, los talleres de Ruta Estratégica de Transformación permitieron involucrar a los actores en un lenguaje propositivo que facili- 
tara pasar de la identificación de problemáticas a alternativas de solución.

Uno de los mayores retos fue la generación de confianza entre los actores participantes del proceso de construcción de la política pública debido a que, por lo general, se crean altas expectativas de ver resultados inmediatos en el momento de su implementación, es por ello que fue importante aclarar que es un proceso que obtiene resultados en el corto, mediano y largo plazo.

De otro lado, se logró que la comunidad se empoderara de las alternativas de solución de sus problemáticas, por lo que se considera serán los principales veedores de las diferentes acciones que se lleven a cabo. También esta dinámica permitió identificar las capacidades de los actores para gestionar las soluciones a sus problemas, pasando de ser dependientes a proactivos.

Además, se considera que la puesta en marcha de las acciones propuestas dentro de las líneas estratégicas, no solo es una oportunidad para solucionar los problemas de empleo, sino para fortalecer la economía local e incentivar los emprendimientos. Asimismo, se considera que este proceso contribuirá a solucionar los diferentes conflictos que se generan alrededor del empleo, al crear canales de comunicación que facilitan que los actores se reconozcan en medio de las diferencias, a fin de lograr acuerdos para el desarrollo de su municipio.

Es importante señalar que la metodología expuesta hace parte de la etapa de formulación, la cual se realizó mediante un proceso de construcción participativa horizontal. Sin embargo, su implementación requiere la realización de un acuerdo municipal que debe ser aprobado por el Concejo Municipal. Este último debe contener los elementos jurídicos acordes con el marco normativo colombiano.

Para ello se recomendó que, de la mano del proceso de aprobación, se realizara un pacto social por el empleo digno y decente en el municipio de Puerto Boyacá, dado que esta política debe ser una apuesta tripartita entre el gobierno local, la empresa y las organizaciones sociales. Esto permitirá que la política sea un proceso inclusivo en el que los actores se empoderen del desarrollo de la implementación. Es importante señalar que si esto no se hace, difícilmente se podrán llevar a cabo los programas y proyectos delimitados en la política pública.

Por último, se considera que la implementación de la política pública de generación de empleo debe ir acompañada del establecimiento de un canal de comunicación que permita que la comunidad de Puerto Boyacá esté informada sobre los avances de los programas y proyectos, y que pueda acceder a información relacionada con las oportunidades de empleo, fortalecimiento productivo, educación y generación de nuevas empresas. El éxito de esta política pública debe ir acompañado de los esfuerzos del Gobierno nacional, departamental y local, de articularse para la realización de programas y acciones delimitadas.

Finalmente, es importante contar con el acompañamiento técnico de las entidades territoriales del nivel central (Ministerio de Trabajo, Ministerio de Comercio Industria y Turismo, DNP, entre otras), para la implementación de los programas y proyectos de la política pública. Esto enmarcado en una 
apuesta por la integración regional con el ánimo de sumar esfuerzos para generar proyectos de alto impacto.

\section{REFERENCIAS}

Albuquerque, F. (s. f.). Desarrollo económico territorial y empleo. Una estrategia para reducir la pobreza y la inequidad social. Madrid: Consejo Superior de Investigaciones Científicas. Recuperado de http://www.bivica.org/upload/desarrollo-inequidad.pdf

Albuquerque, F. (1997). Metodología para el desarrollo económico local. Chile: ILPES-CEPAL.

Centro de Estudios Regionales del Magdalena Medio (CER) (2016). Diagnóstico económico y sociopolítico de Puerto Boyacá (Boyacá). Barrancabermeja: CER.

Centro de Estudios Regionales del Magdalena Medio (CER) (9 de septiembre, 2016). Grupo Focal Concejo Municipal de Puerto Boyacá [Relatoría]. Puerto Boyacá (Boyacá).

Centro de Estudios Regionales del Magdalena Medio (CER) (30 de noviembre, 01, 02 y 03 de diciembre del 2016). Taller de Identificación de Problemáticas cabecera municipal, Puerto Serviez, Muelle Velásquez y Palagua [Relatoría]. Puerto Boyacá (Boyacá).

Centro de Estudios Regionales del Magdalena Medio (CER) (2017). Política pública de generación de empleo de Puerto Boyacá (Boyacá). Barrancabermeja: CER.

Centro de Estudios Regionales del Magdalena Medio (CER) (18 y 25 de enero del 2017). Talleres Rutas de Transformación [Relatoría]. Puerto Boyacá (Boyacá).
Centro de Estudios Regionales del Magdalena Medio (CER) (01 de febrero del 2017). Taller de validación y retroalimentación de la PPGE [Relatoría]. Puerto Boyacá (Boyacá).

Centro de Estudios Regionales del Magdalena Medio (CER) (18 de mayo del 2017). Socialización y entrega del documento de la PPGE [Relatoría]. Puerto Boyacá (Boyacá).

Fundación para el Desarrollo Local y el Fortalecimiento Municipal e Institucional de Centroamérica y el Caribe (DEMUCA) (2009). Guia de herramientas municipales para la promoción del desarrollo económico local. San José de Costa Rica: Demuca.

Godet, M. y Durance, P. (2009). La prospectiva estratégica para las empresas y los territorios (Serie de Investigación 10). Paris: Lipsor.

González, P., Peiró, J. y Bravo, M. (2014). Calidad de vida laboral. En J. Peiró y F. Prieto (eds.). Tratado de psicología del trabajo. Vol. 1. La actividad laboral en su contexto. Madrid: Síntesis.

Keynes, J. (1943). Teoría general de la ocupación, el interés y el dinero. México: Fondo de Cultura Económica.

Ministerio del Interior (s. f.). El enfoque diferencial y étnico en la política pública de víctimas del conflicto armado. Bogotá: Ministerio del Interior.

Morales, J. A. (2012). ¿Qué hay detrás de la maldición de los recursos naturales? Estudio de caso: los paises bajos. Madrid: Instituto Universitario de Desarrollo y Cooperación, Universidad Complutense de Madrid.

Mozas, A. y Bernal, E. (2006). Desarrollo territorial y economía social. Universidad de Jaén. Revista de Economía Pública, Social y Cooperativa, 55, $125-140$

Nolasco (s. f.). Estrategias de enseñanza en educación. Recuperado de https://www.uaeh.edu.mx/scige/ boletin/prepa4/n4/e8.html 
Parsons, W. (2007). Políticas públicas: una introducción a la teoría y la práctica del análisis de politicas públicas. México: Facultad Latinoamericana de Ciencias Sociales (FlACSO).

Plan Nacional de Desarrollo 2014-2018. “Todos por un nuevo país. Paz, equidad y educación”. Bogotá: Gobierno Nacional.

Programa de las Naciones Unidas para el Desarrollo (PNUD) (s. f.). Identificación de las actividades económicas y productivas de mayor desarrollo en el ámbito municipal. Bogotá: PNUD.

Rosanvallon, P. (2007). La contrademocracia. La politica en la era de la desconfianza. Buenos Aires: Manantial.

Ross, M. L. (1999). The Political Economy of The Resource Curse. World Politics, 51, 297-322.

Roth, A. (2002). Politicas públicas. Formulación, evaluación e implementación. Bogotá: Ediciones Aurora.

Sampieri, R. (2014). Metodología de la Investigación (6 ed.). México: McGraw-Hill.
Schuldt, J. y Acosta, A. (2006). Petróleo, rentismo y subdesarrollo, ¿una maldición sin solución? Revista Nueva Sociedad. Democracia y politica en América Latina, 204, 71-89.

Silva, I. (2005). Desarrollo económico local y competitividad territorial. Revista de la CEPAL, 85, 81-100.

Stiglitz J. (2002). El desarrollo no es solo crecimiento del pIB. Revista de Ciencias Sociales, 13, 72-86.

Stiglitz J. (2003). El rumbo de las reformas. Hacia una nueva agenda en América Latina. Revista de la Cepal, 80, 7-37.

Suárez, P. (2011). El servicio público de empleo en Espańa: ensayos desde una perspectiva regional. Oviedo: Universidad de Oviedo. Recuperado de http://www.tdx.cat/bitstream/handle/10803/91066/uov0098Trsc.pdf? sequence $=5$ 
